\title{
Gastric intestinal metaplasia - age, ethnicity and surveillance for gastric cancer
}

\author{
Simko V, Anand N, Ginter E \\ Brooklyn Veteran Medical Center SUNY Downstate Medical Center, Brooklyn, New York. \\ simko.vlado9@gmail.com
}

\begin{abstract}
Aim: Determine the prevalence and distribution of gastric intestinal metaplasia (GIM) in a large cohort of patients subjected to esophagogastroscopy (EGD). Evaluate usefulness of grading the severity of gastritis, GIM and the impact of Helicobacter pylori (HP). Define the population at risk for gastric adenocarcinoma (GC) and assess the value of surveillance.

Methods: In the course of 19 years, we performed 11,600 sequential EGDs in male veterans at Brooklyn, New York. Of all patients, $47 \%$ had EGD only one time while $53 \%$ had EGD repeated, $11 \%$ of these had four or more EGDs. Patients with GIM were matched with equal number of controls with no GI symptoms. All gastric biopsies were processed in one laboratory, using the standardized protocol for histological staining and for grading the severity of epithelial changes.

Results: Of all patients subjected to EGD, 354 (3.05\%) were diagnosed with GIM. Compared to controls, GIM patients were older, $80 \%$ were over 71 . Regarding ethnicity, GIM was $5.4 \%$ more frequent in 177 African Americans than in 159 Caucasians. Distribution of GIM did not differ with respect to age or ethnicity. As many as $6 \%$ of GIM cases were diagnosed with GC. Grading of GIM severity had a predictive value, the average grade of severity in GC was $50 \%$ higher than in non-cancer patients with GIM. Severity of gastritis was also a useful biomarker: patients with GC had more severe gastritis. Surprisingly, HP positivity had no predictive value: HP positive patients had similar distribution of GIM as the HP negative patients. Use of proton pump inhibitors in the past was unknown.

Conclusion: Prevalence of GC in patients with GIM was more than 200 times higher than reported in normal population. Age more than 70 years and African Americans appeared to be at higher risk. Routine EGD and histological diagnosis, with simple grading of severity of epithelial changes provides a useful predictive information. Individuals with upper GI symptoms undergoing EGD with gastric biopsy benefited from routine clinical screening for GC. Patients with higher severity of GIM should enter surveillance (Tab. 1, Fig. 10, Ref. 45). Text in PDF www.elis.sk.

Key words: gastric intestinal metaplasia, gastric adenocarcinoma, gastritis, histological severity, helicobacter pylori, african americans, endoscopy, surveillance.
\end{abstract}

Gastric intestinal metaplasia (GIM) is the replacement of normal gastric glands with an intestinal crypt phenotype. Current theory $(1,2)$ proposes that there is a stepwise progression from chronic atrophic gastritis to GIM (Fig. 1). Dysplasia can arise from a single clone of mutated GIM and further progressively expand $(3,4)$. Conversion of gastric into intestinal type epithelium can be easily detected by histopathology, based on markedly different cellular organization. The extent of GIM can vary from partial intestinalization to a diffuse, in which large portions of the stomach resemble the intestine (5). Several subgroups of GIM are morphologically recognized in relation to the type of mucin (Type I complete and Types II and III, incomplete) $(6,7)$. Sub typing of GIM related to mucin requires special stains, associations between mucin and GC have not been universally recognized (8).

Brooklyn Veteran Medical Center SUNY Downstate Medical Center, Brooklyn, New York

Address for correspondence: V. Simko, MD, PhD, AGAF, FACG, SUNY Downstate Medical Center, 102 Signal Hill Road, Staten Island, NY10301,USA Phone: +7187270955

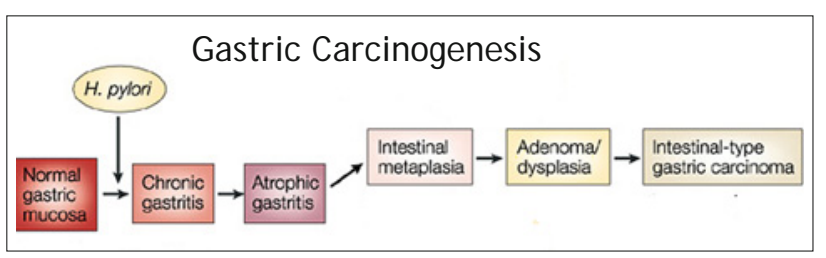

Fig. 1. Progression of gastric epithelial changes: Gastritis, gastric intestinal metaplasia (GIM), dysplasia to intestinal type adenocarcinoma.

Gastric cancer is the second highest cause of malignancy- related deaths worldwide (9). Early stages of GC are usually asymptomatic, diagnosis frequently occurs at an advanced stage, contributing to the high mortality for GC. Although there is a very high incidence of GC in Japan, the reported 5-year survival is relatively high. An intensive population screening results in $50 \%$ of GC being diagnosed at a stage when curative resection is possible (10).

A widely accepted classification of GC distinguished two types (11), the intestinal (more differentiated) and the diffuse (undifferentiated). Intestinal type has malignant cells attempting to form 
glandular structures. Adjacent GIM was also described in $60 \%$ of cases with a diffuse GC (12). Early stage GC can arise in non intestinalized epithelium (13).

Chronic gastritis is an essential link that leads to GC. Activity of gastritis refers to the density of mucosal infiltration by neutrophilic polymorphs (14). The presence of chronic active gastritis and colonization with Helicobacter pylori (HP) indicates a very close association, confirming a tight pathophysiological link (15). There is a strong epidemiological evidence that populations with HP have an increased risk of GC $(16,17)$. GC was reported in 0.1 to $3 \%$ of individuals infected with HP (18). HP infection is classified as a human carcinogen by the World Health Organization (19).

There remains ambivalence on prognostic risk of GC in an HP infected individual. HP colonization is acquired frequently before the age of ten and in absence of antibiotic therapy it persists for life (20). When atrophic gastritis persists for a long time, thinning of the gastric mucosa may diminish or even eradicate the HP (21). By targeting host immune cells, HP can down regulate host responses, maximizing HP persistence (22). HP strains exhibit a high level of genetic diversity (23). Striking difference among HP strains in relation to aggressiveness depends on the presence of a gene that encodes an effector protein (Cag A). This adversely affects gastric epithelial cells, perturbs their cell organization and polarity, impairing the tight junctions between epithelial cells (24). Compared to uninfected subjects, individuals with Cag A positive HP were at considerable risk of GC (25). Tests for different HP strains are in the realm of research.

Eradication of HP reduces the progression of atrophic gastritis but there is little evidence for reversal of $\operatorname{GIM}(26,27)$. It has been estimated that about $50 \%$ of human population is infected with HP (28). Considering high prevalence of HP in populations, some authors expressed doubt if HP alone is the cause of atrophic gastritis and GC (29). HP may just provide an environment that is conducive to carcinogenesis. Even persistent long time colonization of the human stomach usually does not have adverse effects (30). World prevalence of GC does not support the conclusion that individuals with HP have up to 10.9 fold higher chance of developing GC (31).

Further uncertainty in managing HP colonization is related to recent studies that HP negative chronic gastritis may be common (32). Twenty one per cent of patients with histological gastritis tested negative for HP on biopsy from seven gastric sites, by HP culture and HP serology. Previous proton pump inhibitor use was a potential confounding factor. Some authors advocate for HP patients the test-and-treat strategy that avoids the cost and inconvenience of endoscopy, yet it burdens the patient with the expense and side effects of treatment, without documented benefit (33).

Cost effectiveness of endoscopic surveillance of GIM is mostly undocumented. A decision analysis model supported the cost effectiveness of endoscopic surveillance in patients with GIM (34). The objective of present study was to explore and validate the routine clinical diagnostic value of gastric histology, of grading the severity both of gastritis and of GIM and to assess the clinical value of HP diagnosis. Risk of GC related to GIM was also documented in relation to age and ethnic background of patients.

\section{Methods}

In a clinical prospective study lasting 19 years, 11,600 sequential routine EGDs and gastric biopsies were performed in male veteran patients referred for upper gastrointestinal symptoms. To compare age and ethnic distribution, the 354 patients identified with GIM were matched with equal number of non endoscopic controls, with no gastrointestinal or other serious health problems, randomly selected from out patient clinics. Not included were known users of proton pump inhibitors, of antibiotics or NSAIDs, as well as patients undergoing emergent EGD.

This study was performed at the Brooklyn Veteran Medical Center that cares mostly for veterans from southeastern New York City. The project was approved by the Institutional Study Board. All patients signed an informed consent, no individual patient information was revealed before inclusion in the analysis. Endoscopic biopsy was performed primarily in the antrum of the stomach but additional gastric sites were biopsied at the discretion of the experienced supervising endoscopist. A senior gastroenterologist was responsible for the total duration of the project. EGD and the biopsy were done more than once in $53 \%$ of patients, $11 \%$ of patients had four or more EGDs (Fig. 2). Patients who were diagnosed with HP were advised to initiate HP eradication (a proton pump inhibitor and usually two antibiotics, amoxicillin and clarithromycin for 7 to 14 days). EGD was not done to confirm eradication.

Biopsy tissue was immediately immersed in $40 \%$ formaldehyde and submitted to only one pathology department, using standardized staining with H\&E and Giemsa. Uniformity in pathologic diagnosis was assured throughout the study by the same two pathologists, using an established set of diagnostic criteria and terminology. GIM was identified by the presence of mucin containing goblet cells. The extent of GIM was assessed in five grades (Fig. 5), from focal (<5\% goblet cells per power field) to extensive (> $50 \%$ GIM cells) and up to frank dysplasia (grade 5). Histological severity of chronic gastritis was determined by the extent of inflammatory infiltrate and it was graded as absent, mild, moderate

\section{Endoscopic Screening for Gastric Intestinal Metaplasia (GIM)}

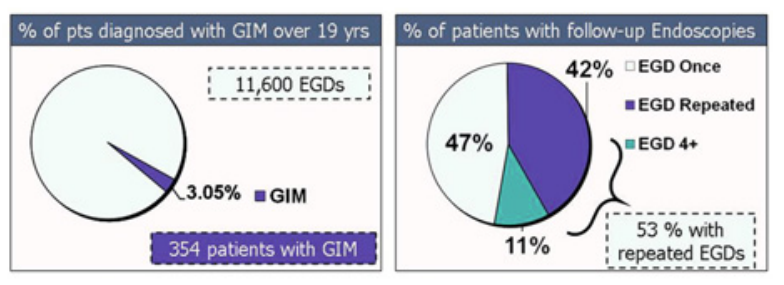

Interval between initial and last EGD was 39.4 months (SD of 34.5 months)

Fig. 2. Over 19 years, in 11,600 upper endoscopies (EGDs) there were 354 (3.05\%) patients diagnosed with GIM at the initial endoscopy. Of these, $47 \%$ had EGD only once, $53 \%$ were endoscoped repeatedly (11\% of them more than four times). 
or severe. This was complemented by the observation and counting of gastric inflammatory nodules in the course of the EGD.

\section{Results}

In the course of 19 years and 11,600 EGDs, 354 patients (3.05\%) were diagnosed with GIM (Fig. 2). In $53 \%$ of those who underwent repeated EGD, the interval between the initial and the last EGD was 39.4 months (SD 34.5 months).

Regarding the age, Figure 3 indicated that the veteran population, both the controls and the GIM patients were older. However, when GIM individuals were compared to the non gastroenterological controls, they were mostly in the $71-80$ years age group, $17 \%$ being older than the controls in this age group. At $81-90$ years, $8 \%$ with GIM were older than the controls. Altogether, 80 $\%$ of patients with GIM were older than 71 years and only $1.4 \%$ were below the age of 50 .

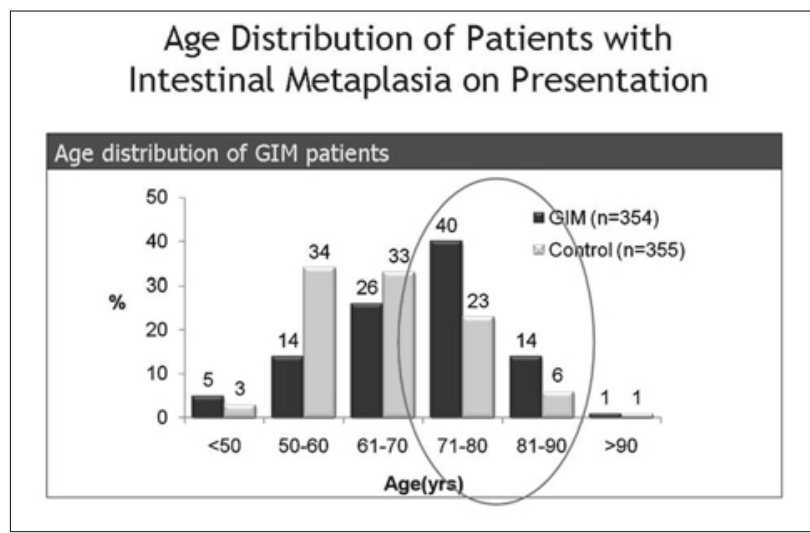

Fig. 3. Compared with 355 non- gastrointestinal controls, patients with GIM were more frequently in the age group $71-90$ years.

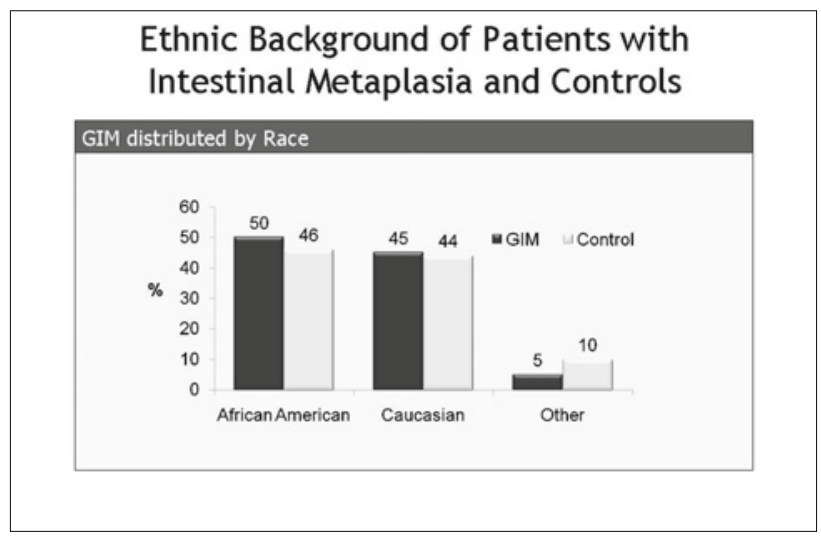

Fig. 4. Compared with non- GI controls, there were more African Americans with GIM. White patients did not show predisposition for GIM.

Ethnic background (Fig. 4) indicated that more patients with GIM (177) were African Americans while 159 were Caucasians (white). GIM was $5.4 \%$ more frequent in African Americans. Compared to the controls, $4 \%$ more African Americans had GIM.

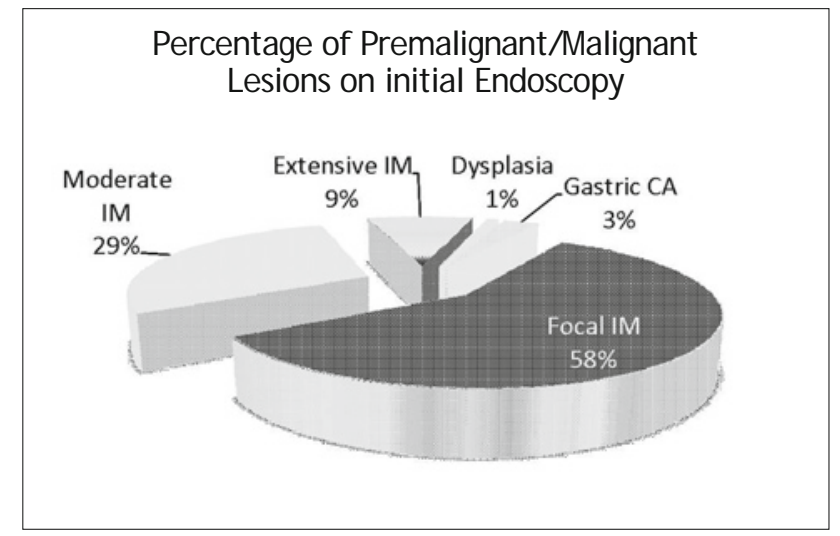

Fig. 5. Severity of GIM on initial endoscopy. Focal (mild) and moderate GIM were present in $87 \%$ of all GIM patients. But $13 \%$ individuals were in the high risk GIM group.

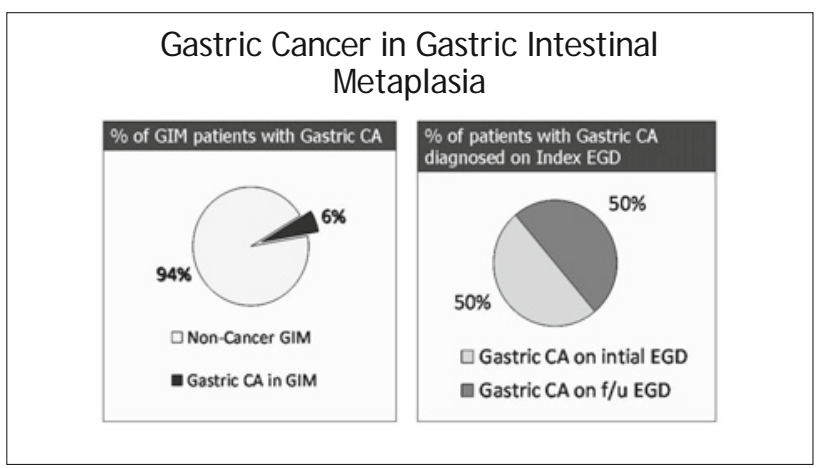

Fig. 6. Of patients with GIM, 6 \% were diagnosed with gastric cancer in the course of the study. When EGD was done repeatedly, the number of cancer cases at baseline examination was doubled.

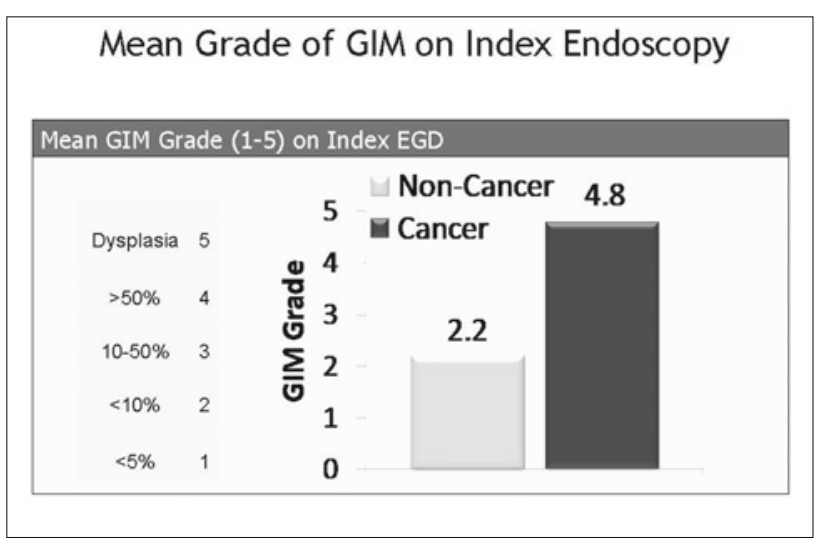

Fig. 7. When severity of GIM was graded 1 - 5 (mild to dysplasia), patients diagnosed with gastric cancer had more than double the severity of GIM.

Other ethnic patients (Hispanic, Asian, American Indian) were under represented in both GIM and in the controls.

Figure 5 indicated the severity of GIM on initial EGD in all 354 patients regardless of age and ethnicity. Most frequent category, in $58 \%$ was focal GIM followed by moderate (29\%), extensive GIM (9\%), dysplasia (1\%) and GC in 11 patients (3\%). When the 


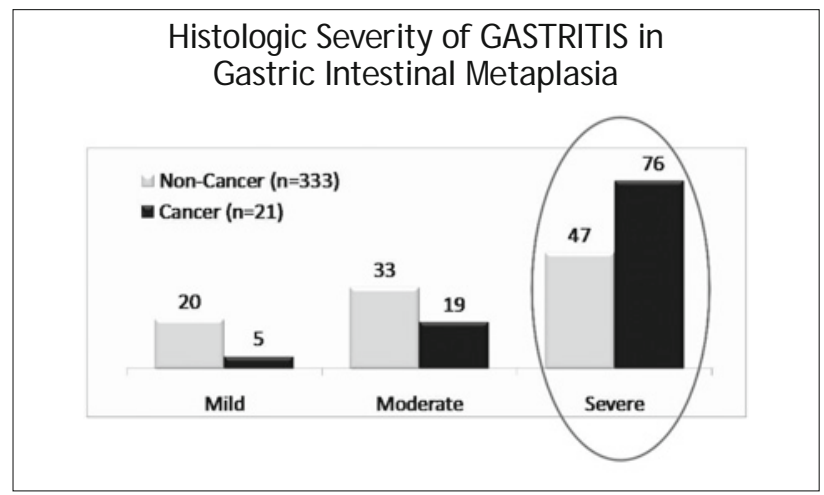

Fig. 8. Percentage of patients with severe gastritis was much higher in gastric cancer patients than in non- cancer GIM

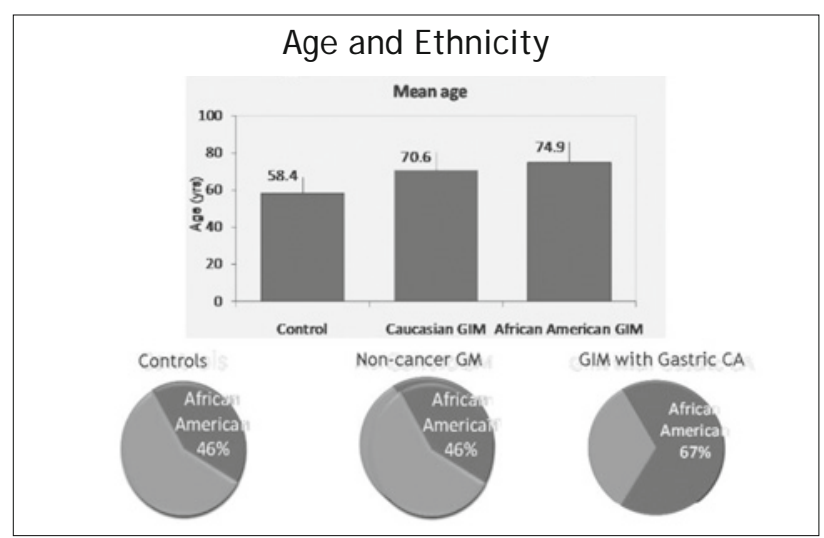

Fig. 9. Age and ethnicity indicate the risk of GIM and gastric cancer. GIM patients were older than non-gastrointestinal controls. While African Americans had similar GIM distribution as other GIM ethnic groups, they were of older age and with higher incidence of cancer.

results of all EGDs, including multiple repeat examinations in patients with higher severity of GIM, were evaluated, the final number of patients with GC increased to $6 \%$, representing 21 patients of the 354 cohort with GIM (Fig. 6). Severity of GIM thus represents risk for GC. When GIM was graded on the scale $1-5$, the average grade of GIM in GC patients was more than $50 \%$ higher (4.8 vs 2.2 in GIM patients with no cancer) (Fig. 7). Distribution of severity of GIM did not differ with respect to age or ethnicity.

Regarding severity of gastritis in GIM (Fig. 8), 38 \% more patients with GIM associated with GC had severe gastritis when compared to non- cancer GIM. Only $24 \%$ of individuals with GC had mild to moderate severity while $53 \%$ of the non- cancer GIM had a lower degree of gastritis. Evaluation of gastric inflammatory

Tab. 1. Percentage of patients with $H$. pylori and breakdown by pathology on initial endoscopy.

\begin{tabular}{lcc}
\hline Pathology & Non $\mathrm{H}$. pylori $\mathrm{n}=201$ & H. pylori $\mathrm{n}=153$ \\
\hline focal IM & $57 \%$ & $58 \%$ \\
moderate IM & $29 \%$ & $31 \%$ \\
extensive IM & $9 \%$ & $8 \%$ \\
dysplasia & $1 \%$ & $1 \%$ \\
gastric ca & $4 \%$ & $2 \%$ \\
\hline
\end{tabular}

nodules visualized at EGD examination did not prove useful: only $20 \%$ of patients with GIM and GC had such nodules.

Figure 9 indicated the age and ethnic distribution related to patients with GIM and in GC, compared to the controls. Patients with GIM had an age on the average 14 years higher than the controls. Regarding ethnicity, African Americans more frequently had GIM compared to Caucasians and $17 \%$ more of them had GIM associated with GC.

Table 1 illustrated the impact of HP diagnosis related to severity of GIM. Of the 354 cases with GIM, 153 (43\%) had HP positive gastric biopsy on the first EGD. Surprisingly, these HP positive patients with GIM had very similar distribution of GIM severity as the HP negative individuals. Only $2 \%$ of HP positive GIM patients had GC while GC was diagnosed in $4 \%$ of HP negative cases.

\section{Discussion}

This study contributed information for diagnostic targeting in patients with gastrointestinal disorders undergoing routine EGD examination. Several markers of risk for GC were identified. Importantly, the prevalence of GC in patients with GIM (at $6 \%$ ) was more than 200 times higher than in the general population.

GIM diagnosed on routine endoscopic gastric biopsy is an important prognostic marker. Of all patients subjected to EGD, $3.05 \%$ had GIM. Histological grading of severity of GIM proved very useful. While most patients (58 \%) had low (focal) severity of GIM, patients with higher severity of GIM were at risk for dysplasia and GC. Progression of GIM severity is a relevant and an important prognostic indicator (Fig. 7). Repeated EGD in patients with higher GIM severity proved useful: while on initial EGD there were $3 \%$ of GIM patients with GC, when EGD was repeated more often, additional $3 \%$ of GIM patients were found with GC. Grading of severity of gastritis also proved useful. GIM patients with GC, had in $74 \%$ a higher severity of gastritis.

Age and ethnic background were additional valuable markers for screening the GIM and GC. Patients with GIM were older than the controls, $80 \%$ were older than 71 . It has been well known that GIM is closely associated with age. This may reflect the time it takes for immune response to gastritis or HP to advance from chronic active gastritis to GIM and dysplasia (15).

Regarding ethnicity, GIM was $5.4 \%$ more frequent in African Americans than in Caucasians. This is somewhat less than in other reports (35), possibly because our patients were veterans and thus of potentially better socioeconomic status, provided with standardized health care quality. African Americans in general are at higher risk for GC and also for other types of malignancy, prostate cancer and liver disease $(36,37)$. This may be related to variability in tumor biology and immune response, in addition to socioeconomic factors and access to health care.

Unexpectedly, in contrast to others, we did not confirm the predictive value of HP positivity as a driving force for severity of gastritis and advanced stage of GIM. In another report, a panel of HP serology markers suggested that in low-income African Americans there is a high prevalence of antibodies against HP 
(38). This would go along with a lower prevalence of acid reflux and Barrett's esophagus in African Americans (39): higher gastric colonization with HP may be responsible for lower acid production and less esophageal reflux. Regarding histological diagnosis of HP, this has good sensitivity and specificity (40).

Another confounding factor may be the HP-negative gastritis (32). Absence of predictive value of HP diagnosis in our study did not contradict the theory of an essential link between HP colonization, chronic gastritis and GIM. Advanced age individuals might have been exposed to HP colonization in many previous life decades. It is doubtful that our HP findings were related to elimination of HP due to atrophic gastritis: gastritis was present and we documented the prognostic value of severity of gastritis as a marker for GIM and GC. The confounding effect of previous undocumented HP suppression with proton pump inhibitors is real, especially when in the past decades this medication has been very liberally administered for esophageal reflux.

The strength of this study is in the large sample of patients and in validating routine diagnostic procedures over the 19 years of duration of the project. Despite using routine and easily reproducible diagnostic methods, valuable results were obtained that can serve as practical guidelines. Data collection and histopathological evaluation was performed only at one site, by a dedicated permanent team. Almost one half of the patients diagnosed with GIM had the EGD performed repeatedly. Grading of histological severity of gastritis and GIM proved effective in assessing progression to GC.

The study also had limitations. It was performed in a selected population of male veterans. The institutional standard of care and quality control were probably above the average obtained at community hospitals in more remote regions. The extent of the project did not allow consistent multiple biopsy mapping at various gastric locations. Our pathologists did not morphologically differentiate, analyzing the mucin, the type of GIM (I to III). The laboratory did not have the capacity to do such analysis and the value of association of subtypes of GIM with the risk of GC has not been fully confirmed (8). Also, if there was a capacity to do so, information on HP colonization would be stronger if HP strains (e.g. Cag A positivity) were included. Data on HP colonization in our patients might have been modified by medications used prior to entering the study (potential bias affecting many other studies). Detailed historic data on life style (smoking, alcohol, socioeconomic status) to associate with GIM were not collected due to the extent of the project.

Endoscopic surveillance for GIM has not been previously adequately evaluated $(41,42)$. This project contributes to the practical objective regarding patients`screening and surveillance for GIM as a biomarker and potential precursor of GC. Population at risk was identified as individuals with upper gastrointestinal symptoms past the age of 70 and especially African Americans. Grading of histological severity of gastritis and GIM has a predictive value and indicates the need for the frequency of endoscopic surveillance. This may not be required in a low risk individual. Due to multifocal nature of GIM epithelial changes, surveillance should include gastric biopsy mapping at multiple locations. The use of proton pump inhibitors and antibiotics should be monitored in fu- ture studies to avoid false negative HP findings Specialized referral centers in geographic areas with high prevalence of GC may also employ novel endoscopic diagnostic procedures, confocal laser endomicroscopy, narrow band imaging and chromoendoscopy $(43,44,45)$. However, clinically useful results were derived here from easily available diagnostic techniques.

In conclusion,_a large prospective endoscopic screening over 19 years and 11,600 procedures identified 354 patients with GIM. Histological severity of gastritis and of GIM was associated with the risk of GC. Initial endoscopy revealed $3 \%$ of patients with GIM to have GC, but when subjects at risk were examined repeatedly, additional $3 \%$ had GC. Prevalence of GC in patients with GIM was thus over 200 times higher than reported in the general population. GIM was more frequent in patients older than 70 and in African Americans. Endoscopic screening is justified in such subjects who present with upper GI symptoms. There is a long lead time in progression of GIM to GC. When GIM is associated with higher degree of gastritis, endoscopic surveillance offers an opportunity for prevention and for timely intervention to counter the asymptomatic progression of incipient GC to advanced stages.

\section{References}

1. Correa P. A human model of gastric carcinogenesis. Cancer Res 1988; 48: 3554 - 3560.

2. Correa P, Shiao YH. Phenotypic and genotypic events in gastric carcinogenesis. Cancer Res 1994; 54: S 1941 - S 1943.

3. Gutierez- Gonzalez L, Graham T.A, Rodriguez- Justo M et al. The Clonal Origins of Dysplasia from Intestinal Metaplasia in the Human Stomach. Gastroenterology 2011; 140: 1251 - 1260.

4. de Dombal FT, Price AB, Thomson $\mathrm{H}$ et al. The British Society of Gastroenterology early gastric cancer/ dysplasia survey: an interim report. Gut 1990; 31: 115 - 120 .

5. Mills JC, Shivdasani RA. Gastric epithelial stem cells. Gastroenterology 2011; 140: $412-424$.

6. Correa P, Piazuello B, Wilson KT. Pathology of Gastric Intestinal Metaplasia: Clinical Implications. Am J Gastroenterol 2010; 105: 493 -498 .

7. Rokkas T, Filipe MI, Sladen GE. Detection of an increased incidence of early gastric cancer in patients with intestinal metaplasia type III who are closely followed up. Gut 1991; 32: 1110 - 1113.

8. Kang KP, Lee HS, Kim N et al. Role of intestinal metaplasia subtyping in the risk of gastric cancer in Korea. J Gastroenterol Hepatol 2009; 1: $140-148$.

9. Pankin DM, Bray F, Ferlay J T et al. Global cancer statistics, 2002. CA Cancer J Clin 2005; 55: 74 - 104.

10. Axon A. Symptoms and diagnosis of gastric cancer at early curable stage. Best Pract Res Clin Gastroenterol 2006; 20: 697 - 708.

11. Lauren P. The two histological main types of gastric carcinoma: diffuse and so-called intestinal type carcinoma. Acta Pathol Microbiol Scand 1965; 64: 31 - 49 .

12. Boussiountas A, Li H, Liu J et al. Distinctive patterns of gene expression in premalignant gastric mucosa and gastric cancer. Cancer Res 2003; 63: $2569-2577$. 
13. Park do Y, Srivastava A, Kim GH et al. Adenomatous and foveolar gastric dysplasia: distinct patterns of mucin expression and background intestinal metaplasia. Am J Surg Pathol 2008; 32: 524 - 533.

14. Dixon MF, Genta RM, Yardley JH et al. Classification and grading of gastritis: the updated Sydney system. Am J Surg Pathol 1996; 120: $1161-1181$.

15. Sonnenberg A, Lash RH, Genta RM. A National Study of Helicobacter pylori Infection in Gastric Biopsy Specimens. Gastroenterology 2010; 139: $1894-1901$.

16. Huang JQ, Sridhar S, Chen $Y$ et al. Meta-analysis of the relationship between Helicobacter pylori seropositivity and gastric cancer. Gastroenterology 1998; 114: 1169 - 1179.

17. Helicobacter and Cancer Collaborative Group. Gastric cancer and Helicobacter pylori: a combined analysis of 12 case control studies nested within prospective cohorts. Gut 2001; 49: 347 - 353.

18. Uemura N, Okamoto S, Yamamoto $S$ et al. Helicobacter pylori infection and the development of gastric cancer. New Engl J Med 2001; 345: $784-789$.

19. Infection with Helicobacter pylori. In: IARC Working Group on the Evaluation of Carcinogenic Risks to Humans. International Agency for Research on Cancer, 61, Lyon France 1994, 177 - 240.

20. Perry S, de la Luz Sanchez M, Yang S et al. Gastroenteritis and transmission of Helicobacter pylori infection in households. Emerg Infect Dis 2006; 12: $1701-1708$.

21. Karnes WE Jr, Samloff IM, Sivrala M et al. Possible serum antibody and negative tissue staining for Helicobacter pylori in subjects with atrophic body gastritis. Gastroenterology 1991; 101: 167 - 174.

22. Cover TL, Blaser MJ. Helicobacter pylori in Health and Disease. Gastroenterology 2009; 136: 1863 - 1867.

23. Blaser MJ, Berg DE. Helicobacter pylori gastric diversity and risk of human disease. J Clin Invest 2001; 107: 767 - 773.

24. Censini S, Lange $\mathbf{C}$, Xiang $\mathbf{Z}$ et al. cag, a pathogenicity island of Helicobacter pylori, encodes type I-specific and disease-associated virulence factors. Proc Natl Acad Sci USA 1996; 93: 14648 - 14653.

25. Parsonnet J, Friedman GD, Orentreich $\mathbf{N}$ et al. Risk for gastric cancer in people with Cag A positive or Cag A negative Helicobacter pylori infection. Gut 1997; 40: 297 - 301.

26. Leung WK, Lin SR, Ching JY et al. Factors predicting progression of gastric intestinal metaplasia: results of a randomized trial of Helicobacter pylori eradication. Gut 2004; 53: 1244 - 1249.

27. Watazi J, Das KK, Amenta PS et al. Effect of eradication of Helicobacter pylori on the histology and cellular phenotype of gastric intestinal metaplasia. J Clin Gastroenterol Hepatol 2008; 6: 409 - 417.

28. De Luca A, Iaquinto G. Helicobacter pylori and gastric diseases: a dangerous association. Cancer Lett 2004; 213: 1 - 10.

29. Crew UKD, Neugut AI. Epidemiology of gastric cancer. World J Gastroenterol 2006; 12: 354 - 362.
30. Algood HM, Cover TL. Helicobacter pylori persistence: an overview of interaction between H. pylori and host immune defenses. Clin Microbiol Rev 2006; 19: 597 - 613.

31. Kim N, Park RY, Cho SI et al. Helicobacter pylori infection and development of gastric cancer in Korea: long term follow up. J Clin Gastroenterol 2008; 42: 448 - 454.

32. Nordenstedt H, Graham DY, Kramer JR et al. Helicobacter pylorinegative Gastritis: Prevalence and Risk Factors. Am J Gastroenterol 2013; 108: $65-71$.

33. Lassen AT, Pedersen FM, Bytzer P et al. Helicobacter pylori test- and -eradicate versus prompt endoscopy for management of dyspeptic patients: a randomized trial. Lancet 2000; 356: 455 - 460.

34. Hassan C, Zullo A, Di Giulio E et al. Cost effectiveness of endoscopic surveillance for gastric intestinal metaplasia. Helicobacter 2010; 15: $221-226$.

35. Jemal A, Siegel R, Ward E et al. Cancer statistics, 2007. CA Cancer J Clin 2007; 57: 43 - 66.

36. Iha AK, Fisher ES, Li Z et al. Racial trends in the use of major procedures among the elderly. New Engl J Med 2005; 353: 683 - 691.

37. Price AL, Butler J, Patterson N et al. Discerning the ancestry of European Americans in genetic association studies. PLoS Genet 2008; 4: e 236.

38. Epplein M, Signorello LB, Wei Zheng et al. Race, African ancestry and Helicobacter pylori infection in a low-income United States population. Cancer Epidemiol Biomarkers Prev 2011; 5: 826 - 834.

39. Abrams JA, Fields S, Lightdale CJ. Racial and Ethnic Disparities in the Prevalence of Barrett's Esophagus among Patients Who Undergo Upper Endoscopy. Clin Gastroenterol Hepatol 2008; 6: 30 - 34.

40. Mc Coll KEL. Helicobacter pylori infection. New Engl J Med 2010; 362: $1597-1604$.

41. Sonnenberg A, Amorosi SL, Lacey MJ et al. Pattern of endoscopy in the United States- analysis of data from the Center for Medicare and Medicaid Services and the National Endoscopic Database. Gastrointest Endosc 2008; 67: 489 - 496.

42. Hirota WK, Zuckerman MJ, Adler DG et al. ASGE guideline: the role of endoscopy in the surveillance of premalignant conditions of the upper GI tract. Gastrointest Endosc 2006; 63: 570 - 580.

43. Samarasena J, Dhillon R, Yosuke $\mathbf{N}$ et al. In vivo Diagnosis of Gastric Intestinal Metaplasia Using Probe Based Confocal Laser - Induced Endomicroscopy. Am J Gastroenterol 2012; 107: S 62.

44. Tahara T, Shibata T, Nakamura M et al. Gastric mucosal pattern by using magnifying narrow-band imaging endoscopy clearly distinguishes histological and serological severity of chronic gastritis. Gastrointest Endosc 2009; 70: $246-253$.

45. Dinis- Ribeiro M, da Costa-Pereira A, Lopes C et al. Magnification chromoendoscopy for the diagnosis of gastric intestinal metaplasia and dysplasia. Gastrointest Endosc 2003; 57: 498 - 504.

Received July 31, 2013. Accepted March 15, 2014. 